\title{
Validity and internal consistency of the French version of the revised Skin Management Needs Assessment Checklist in people with spinal cord injury
}

\author{
Anthony Gélis ${ }^{1,2}$ - Arnaud Dupeyron ${ }^{3,4}$ - Jean Pierre Daures ${ }^{5}$ - David Goossens ${ }^{6} \cdot$ Dominique Gault $^{7}$. \\ Jean Paul Pedelucq ${ }^{8} \cdot$ Michel Enjalbert $^{9} \cdot$ Eric Maupas $^{10} \cdot$ Paul Kennedy $^{11} \cdot$ Charles Fattal $^{12}$. \\ The French SMNAC Group
}

Received: 8 February 2018 / Revised: 6 April 2018 / Accepted: 16 April 2018 / Published online: 12 June 2018

(c) International Spinal Cord Society 2018

\begin{abstract}
Study design Cross-sectional psychometrics study.

Objectives To determine the construct validity and internal consistency of the revised Skin Management Needs Assessment Checklist (revised SMnac).

Setting Six spinal cord rehabilitation centers.

Methods One-hundred and thirty-two community-dwelling individuals with spinal cord injury (SCI) were included. Construct validity was assessed by a Spearman's rank correlation coefficient between the revised SMnac and several questionnaires: Rosenberg Self-Esteem Scale, Ways of Coping Questionnaire, Hospital Anxiety and Depression Scale (HADS), Braden scale; or clinical variables: educational level, presence of a pressure ulcer (PU), history of multiple PUs, time since injury, and pain.

Results The study evidenced construct validity with a fair to moderate correlation coefficient between the revised SMnac and Rosenberg scale $\left(r_{\mathrm{s}}=0.25 ; p=0.03\right)$, active coping $\left(r_{\mathrm{s}}=0.29 ; p=0.001\right)$, HADS $\left(r_{\mathrm{s}}=-0.43 ; p<0.0001\right)$, and time since injury $\left(r_{\mathrm{s}}=0.49 ; p<0.0001\right)$. The presence of PU and history of multiple PUs were strongly correlated with the revised SMnac score (respectively, $p=0.01$ and 0.001). Internal consistency was excellent $(\alpha=0.907)$.

Conclusion These results show that the revised SMnac is a valid tool to assess PU self-management in individuals with SCI. Further studies are needed to assess the revised SMnac's responsiveness to change.
\end{abstract}

\section{Introduction}

Pressure ulcer (PU) is a common complication in people with spinal cord injury (SCI). According to the studies, the

Anthony Gélis

a.gelis@ propara.fr

1 Centre de Rééducation Neurologique Mutualiste Propara, Parc Euromédecine, Montpellier, France

2 Laboratoire Epsylon, Université de Montpellier, Montpellier, France

3 Département de Médecine Physique et de Réadaptation, Centre Hospitalo-Universitaire Caremeau, Nîmes, France

4 LaboratoireEuromov, Université de Montpellier, Montpellier, France

5 Institut Universitaire de Recherche Clinique, Montpellier, France prevalence varies between 30 and $60 \%$ in persons with SCI [1, 2], and thus represents a major public health challenge both on a medical level with high rate of associated complications and on an economic level such as

6 Centre de rééducation de la Tour de Gassies, Bruges, France

7 Centre de rééducation Clemenceau, Strasbourg, France

8 Centre de Rééducation Fonctionnelle de Kerpape, Ploemeur, France

9 Centre de Rééducation Bouffard Vercelli, Cerbere, France

10 CentreMutualiste de rééducation, Albi, France

11 Department of Clinical Psychology, National Spinal Injuries Centre, Stoke Mandeville Hospital, Buckinghamshire, UK

12 Centre de reeducation fonctionnelle La Chataigneraie, Menucourt, France 
extended duration of hospital stays or multiple hospital stays.

In people with SCI, PU prevention is based on adequate prevention devices choice (wheelchair cushion, and air mattresses), nutrition, and adopting skin protection behaviors [3, 4]. Acquiring these skin protection behaviors starts at the initial care management phase, during that adjustment period with SCI's physiological, psychological, and social consequences. Healthcare teams are initially in charge of preventing PU occurrence, yet they progressively transfer this self-care competency to the persons with SCI, using an educational-based approach adapted to each person [5]. This learning process will then continue at home, sometimes for several years, according to PU occurrence and the support given by healthcare teams.

As soon as an educational strategy is defined for people with SCI, it is essential to have the necessary means to evaluate the impact of this strategy not only on patients' knowledge but also on self-implemented prevention measures. Most studies in the literature focused on evaluating the impact of a standardized therapeutic education on PU recurrence; patients' knowledge in terms of preventive measures only came as a secondary assessment [6, 7]. Questionnaires used to evaluate patients' knowledge were designed within the framework of these studies and their psychometric qualities are unknown.

The Skin Management Needs Assessment Checklist (SMnac), a self-administered questionnaire, is highly relevant because it was designed to focus on assessing patient's knowledge and self-reported prevention measures in terms of skin lesions [8]. It includes 12 questions divided into three different categories: "Skin checks," "preventing pressure ulcers," and "preventing wounds". It corresponds to the Skin chapter of the NAC (Needs Assessment Checklist) [9], which is a self-administered questionnaire listing the self-management objectives of people with SCI. This questionnaire was validated in the English language. The SMnac validation in the English language was conducted on 317 people with SCI [8] and reported good internal consistency (Cronbach's $\alpha=0.85$ ), as well as a good responsiveness to change. The SMnac test-retest reproducibility was $0.90[9,10]$.

The revised SMnac version was elaborated during translation and trans-cultural adaptation in the French language. For the revised version, six new items were added focused on detecting the early-onset of a PU by skin palpation, on evaluating the increased risk due to time spent in a wheelchair or certain sports practices, as well as life habits (smoking, nutrition). It also includes guidelines on how to react faced with a lingering redness of the skin, or checking PU prevention equipment. We also refined the presentation of the score that was repeated for each item to facilitate the reading and comprehension for the person filling out the questionnaire. This new version needs to be evaluated for its psychometric qualities (reproducibility, validity, and responsiveness to change). A reproducibility study on a first sample of 138 people with SCI was previously reported [11].

The objective of this study was to assess the validity and internal consistency of the Revised SMnac scale.

\section{Methods}

Inclusion criteria were (1) people with traumatic or nontraumatic SCI (2) age $>18$ years. Exclusion criteria were (1) severe cognitive disorders, (2) severe psychiatric disorders, and (3) poor understanding of the French language. Upon inclusion each participant signed an informed consent form. The study protocol was approved by the Ethical Committee of Southern France.

\section{Materials}

\section{Social criteria}

Educational attainment was defined as the highest level achieved in the French educational system [12].

\section{Clinical criteria}

Three clinical criteria were chosen for the construct validity. Time since injury (in months), the presence of a PU at the time of the study, and history or recurrent PU, defined by the presence of two different Stage 2 (or above) PU over a 3 -year period [13].

\section{Rosenberg Self-esteem Scale}

It is a 10-item self-administered questionnaire to evaluate self-esteem. It has two components with five questions each for positive and negative self-esteem. Each item is scored from 1 to $4(1=$ do not agree at all and $4=$ completely agree). The total score ranges from 10 to 40 . A high score indicates a high self-esteem level. The validity in persons using a wheelchair was conducted [14]. Internal consistency was good (Cronbach's $\alpha=0.86$ ) and the factor analysis confirmed the one-dimensional nature. This scale was validated in the French language [15].

\section{Way of Coping Checklist}

It is a 21-item self-administered questionnaire to assess the coping strategies that people use in stressful daily life situations [16]. It has three dimensions (seeking social support, problem solving, and avoidance), with 6,8 , and 7 items, respectively. Each item is scored from 1 (not used) to 
4 (used a lot). The score of each category reflects the type of coping strategy preferentially used. The internal consistency of each dimension was high (Cronbach's $\alpha=0.87,0.81$, and 0.73 , respectively).

\section{Hospital Anxiety and Depression Scale}

Hospital Anxiety and Depression Scale (HADS) is a 14item self-administered questionnaire to evaluate anxiety and depression. It has two components with seven questions. It uses a four-level quotation (i.e., ranging from 0 to 3). The score for each component ranges from 0 to 21 . A higher score indicates depression or anxiety. It is a scale commonly used in clinical practice and validated in the French language and its psychometric properties were largely evaluated in people with SCI [17-19].

\section{The Braden Scale for Predicting Pressure Sore Risk}

The Braden Scale for Predicting Pressure Sore Risk is a 6item scale to assess PU risk. The items are sensory perception, mobility, activity, moisture, nutrition, friction, and shear [20]. Each item is scored from 1 to 4, except for shear, scored from 1 to 3 . The Braden Scale is scored from 6 to 23 points, and the critical cutoff for diagnosing risk across a variety of settings and populations is 18 [21]. A higher score indicates a low PU risk. The interclass correlation coefficient over 1 year was 0.90 ( $95 \%$ confidence interval (CI): $0.8-0.92$ ) and 0.88 (95\% CI: 0.85-0.91). This scale is one of the most validated in the SCI population [22].

\section{The revised SMnac}

The revised SMnac is the French version of the SMnac. Its development was previously reported [11]. It is a 19-item self-administered questionnaire spread out over three categories (skin check, preventing PU, preventing wounds) with 4,11 , and 4 items, respectively. Each item is scored from 0 to 3 ( $0=$ completely dependent, never does to $3=$ completely independent, always does or instructs someone to). A higher score indicates a high level of selfreported PU knowledge and prevention practice. The total score is expressed in percentage. The test-retest reproducibility is excellent (intraclass correlation coefficient: 0.899).

\section{Protocol}

The validity study was conducted on a sample of people with SCI meeting the inclusion criteria. The investigators delivered clear and precise oral information on the study protocol and its objectives and collected the informed signed consent form for each participant.

After collecting the clinical and sociodemographics data, the investigator handed out the self-assessment questionnaires to participants: Revised SMnac, HADS, Way of Coping Check (WCC), and the self-esteem Rosenberg scale, and offered the help of a third party to fill out answers if the participant was unable to do so.

\section{Psychometric analysis}

Construct validity is apparent when an instrument behaves as would be expected in relation to another measurement and refers to the ability of a scale to differentiate subjects in regards to certain characteristics known to the disease process or injury in ways consistent with convergent or divergent hypotheses. A convergent hypothesis means that we expect a correlation between the two variables, and a divergent hypothesis means that we do not expect a correlation.

In the current study, the following seven convergent hypotheses were assessed. The first convergent hypothesis concerned time since injury: if people with SCI learn to better manage the risk of skin damage with time and experience, there in fact exists a correlation with the revised SMnac. The second and third hypotheses concerned the presence of a PU at the time of the study, or the presence of recurrent ulcers: since persons learn from their current and past experiences, they should have a higher SMnac score than persons who never had a PU. The fourth and fifth convergent hypotheses concerned psychological variables: persons with a low self-esteem or presenting with depression exhibit poorer skin prevention behaviors [23, 24]. The sixth and seventh convergent hypotheses related to coping strategies: persons with active coping strategies show more adapted health-related behaviors. Conversely, persons that preferentially use passive coping strategies exhibit at-risk behaviors for skin damage prevention [25].

Three divergent hypotheses were evaluated: the absence of correlation with pain at the time of scale administration, regardless of pain location or type of pain, assessed with a visual analog scale; the absence of correlation with the participants' educational attainment, evaluated by the Poitrenaud score and the absence of correlation with the PU risk measured by the Braden Scale. The Braden Scale evaluates the physiological or physical dimensions of the PU risk, which are on a conceptual level quite distanced from participants' knowledge and health-related behaviors.

Internal consistency refers to the general agreement between multiple items of a scale or a subscale to measure the given construct. A high internal consistency indicates that items evaluating a same concept generate similar scores [26]. 


\section{Statistical analysis}

\section{Construct validity}

The construct validity was evaluated via different convergent and divergent hypotheses using a univariate analysis [27]. For categorical variables we performed $\chi^{2}$ independence tests. For quantitative variables, the Spearman's correlation coefficient $(\rho)$ was computed [28]. Spearman's $\rho$ can help evaluate the construct validity with other scales or quantitative measurement tools. Calculating a correlation coefficient is only the first step in the analysis of a relationship between two variables. It is at most an exploratory step that must be validated by a test confirming that the relationship is significant. When the distribution of variables was normal, an analysis of variance was performed. If the distribution was abnormal, non-parametric tests were used (Mann-Whitney $U$ test for two samples and the Kruskall-Wallis test for more than two samples). A correlation coefficient was deemed excellent $(0.8<\rho \leq 1)$, good $(0.6<\rho \leq 0.8)$, moderate $(0.4<\rho \leq 0.6)$, weak $(0.2<$ $\rho \leq 0.4)$, or poor $(0<\rho \leq 0.2)$. The correlation hypothesis must be validated by the appropriate statistical significance test [28].

\section{Internal consistency}

Internal consistency was evaluated with Cronbach's $\alpha$ and deemed very good $(0.91<\alpha \leq 1)$, good $(0.71<\alpha \leq 0.90)$, moderate $(0.51<\alpha \leq 0.70)$, weak $(0.31<\alpha \leq 0.5)$, or poor $(0<\alpha \leq 0.30)$. It has been shown that internal consistency within a single dimension should be quite high $(\alpha>0.70)$, but that the coefficient should not be closed to 1, otherwise it suggests that several items are redundant and evaluate the same thing [29]. The correlation of dimensions between themselves was evaluated with Spearman's correlation coefficient $(\rho)$, with the same interpretation scale previously described [28].

\section{Results}

\section{Participants}

The study was conducted on a sample of 132 communitydwelling participants (Table 1). The main demographic and clinical characteristics of participants are summed up in Table 1.

\section{Construct validity}

Construct validity was evaluated via seven convergent hypotheses and three divergent hypotheses (Table 2).
Table 1 Clinical and demographics characteristics of the persons with SCI included in the study

\begin{tabular}{|c|c|}
\hline Characteristics & $N=132$ \\
\hline \multicolumn{2}{|l|}{ Demographics } \\
\hline Age (year) & $\begin{array}{l}45.9 \pm 14.9 \\
\text { (range: } 19-82 \text { ) }\end{array}$ \\
\hline \multicolumn{2}{|l|}{ Sex } \\
\hline Men & $99(75)$ \\
\hline Women & $33(25)$ \\
\hline Weight (kg) & $\begin{array}{l}70.4 \pm 14 \\
\text { (range: } 37-114)\end{array}$ \\
\hline Height (m) & $\begin{array}{l}1.73 \pm 0.09 \\
\text { (range: } 150-197 \text { ) }\end{array}$ \\
\hline BMI $\left(\mathrm{kg} \mathrm{m}^{-2}\right)$ & $23.9 \pm 3.7$ \\
\hline \multicolumn{2}{|l|}{ Lesion characteristics } \\
\hline $\begin{array}{l}\text { Age at the time } \\
\text { of injury (years) }\end{array}$ & $\begin{array}{l}38 \pm 16 \\
\text { (range: } 10-82 \text { ) }\end{array}$ \\
\hline $\begin{array}{l}\text { Time since injury } \\
\text { (years) }\end{array}$ & $\begin{array}{l}7.8 \pm 10 \\
\text { (range: } 0.1-58 \text { ) }\end{array}$ \\
\hline \multicolumn{2}{|l|}{ Lesion level } \\
\hline Cervical & $48(36)$ \\
\hline \multicolumn{2}{|l|}{ lower back } \\
\hline \multicolumn{2}{|l|}{ AIS } \\
\hline A & $84(64)$ \\
\hline $\mathrm{B}$ & $17(13)$ \\
\hline $\mathrm{C}$ & $16(12)$ \\
\hline $\mathrm{D}$ & $15(11)$ \\
\hline \multicolumn{2}{|l|}{ Etiology } \\
\hline Traumatic & $103(78)$ \\
\hline Non-traumatic & $29(19)$ \\
\hline \multicolumn{2}{|l|}{ Skin characteristics } \\
\hline Braden (6-23) & $\begin{array}{l}15.8 \pm 3 \\
\text { (range: 9-23) }\end{array}$ \\
\hline \multicolumn{2}{|l|}{ Pressure ulcer } \\
\hline \multicolumn{2}{|l|}{ Number } \\
\hline 0 & $85(65)$ \\
\hline 1 & $33(25)$ \\
\hline 2 and more & $14(10)$ \\
\hline
\end{tabular}

Results are presented in mean \pm standard deviation or $N(\%)$

AIS ASIA Impairment Scale, BMI body mass index

Convergent hypotheses between a high SMnac score and the presence of a PU or history of recurrent PUs were verified ( $p=0.01$ and $p=0.001$, respectively). The convergent hypothesis between time since injury and a high SMnac score was also established $(\rho=0.49, p<0.0001)$.

We formulated four convergent hypotheses with psychological variables. The convergence hypotheses between a low SMnac score and the presence of depression $(\rho=$ $-0.43, p<0.0001)$ or low self-esteem $(\rho=0.25, p=0.03)$ 
Table 2 Construct validity of the revised SMnac

\begin{tabular}{|c|c|c|}
\hline & \multicolumn{2}{|c|}{ Revised SMnac score } \\
\hline & $\begin{array}{l}\text { Correlation } \\
\text { (Spearman) }\end{array}$ & Significanc \\
\hline \multicolumn{3}{|l|}{ Convergent hypotheses } \\
\hline Time since injury & 0.49 & $<0.0001^{\mathrm{a}}$ \\
\hline Rosenberg Scale & 0.25 & $0.03^{\mathrm{a}}$ \\
\hline \multicolumn{3}{|l|}{ WCC } \\
\hline Passive coping & -0.16 & $0.43^{\mathrm{a}}$ \\
\hline Active coping & 0.29 & $0.001^{\mathrm{a}}$ \\
\hline Recurrent pressure ulcers & NA & $0.001^{\mathrm{b}}$ \\
\hline Presence of a pressure ulcer & NA & $0.01^{\mathrm{b}}$ \\
\hline HADS & -0.43 & $<0.0001^{\mathrm{a}}$ \\
\hline \multicolumn{3}{|l|}{ Divergent hypotheses } \\
\hline Braden scale & 0.02 & $0.98^{\mathrm{a}}$ \\
\hline $\begin{array}{l}\text { Educational attainment } \\
\text { (Poitrenaud) }\end{array}$ & NA & $0.37^{\mathrm{b}}$ \\
\hline Presence of pain & NA & $0.51^{\mathrm{b}}$ \\
\hline
\end{tabular}

WCC Way of Coping Checklist, HADS Hospital Anxiety and Depression Scale, NA not applicable

${ }^{\text {a}}$ Kruskall-Wallis

${ }^{\mathrm{b}}$ Mann-Whitney $U$ test

were validated. There was a convergence between the active coping strategies and high SMnac score $(\rho=0.29, p=$ 0.001 ). The convergent hypothesis between the passive coping strategies and low SMnac score was not validated $(\rho$ $=-0.16, p=0.43$ )

The three divergent hypotheses were validated: there was no relationship between the SMnac score and the risk of PU $(\rho=0.02, p=0.98)$, educational attainment $(p=0.37)$, or presence of pain $(p=0.51)$ as measured with the Braden Scale.

\section{Internal consistency}

The internal consistency of the global scale was excellent, evaluated at 0.90 with the Cronbach's $\alpha$ (Table 3). Internal consistencies of the subscales "skin check," "preventing the onset of PU," or "preventing the onset of a wound" were, $0.78,0.84$, and 0.64 , respectively. Discarding three items altered slightly the internal consistency of the scale to 0.91 . These items were "Do you change positions in bed," "Do you position your pillows correctly in your bed?", and "Do you cut your nails as you were instructed to before the onset of ingrown toe nails?" These items did not seem to assess the same concepts than the others, but they did not have a major impact on the scale's internal consistency and discarding them did not seem clinically relevant to us. Correlations between the SMnac subscales were good (Table 4).
Table 3 Internal consistency for the Revised SMnac and its subscales

Cronbach's $\alpha$ coefficient

\begin{tabular}{ll}
\hline SC & 0.78 \\
PPU & 0.84 \\
PW & 0.64 \\
Revised & 0.90 \\
SMnac & \\
\hline
\end{tabular}

$S C$ skin check, $P P U$ preventing pressure ulcer, $P W$ preventing wound

Table 4 Spearman correlation of the revised SMnac subscales

\begin{tabular}{llll}
\hline Subscale & SC & PPU & PW \\
\hline SC & 1 & & \\
PPU & 0.73 & 1 & \\
PW & 0.60 & 0.72 & 1 \\
\hline
\end{tabular}

$S C$ skin check, $P P U$ preventing pressure ulcer, $P W$ preventing wound

\section{Discussion}

This publication reports the validity study of the French revised SMnac on a sample of people with acute and community-dwelling SCI. We used construct validity methodology to evaluate the validity. The general conclusion drawn from the seven convergent hypotheses formulated was that the correlation level was low to moderate. The correlation level observed was most likely linked to the fact that no hypothesis compared the SMnac to another behavioral scale, since no other scale exists in the French language for people with SCI. Thus, we needed to elaborate convergent or divergent hypotheses positioned in a theoretical framework of health psychology and evaluating via experiments the putative relationships between psychological variables (depression, coping, self-esteem) and behavioral and cognitive variables assessed by the SMnac. Furthermore, there were numerous factors involved in the construction of knowledge and health behaviors, and the statistical analysis used for convergent hypotheses is a univariate one. As such we did not expect a high correlation level, and we focused rather on the degree of significance for the relationship. The most important result in the hypotheses formulated was the correlation between the SMnac and the presence of a PU or history of recurrent PU. The scale does assess a concept related to the PU, in light of improving knowledge and prevention practices based on the participants' experience with PU.

All divergent hypotheses were validated. The absence of correlation with the Braden's Scale promotes the fact that the "behavioral" risk was not related to the 
"physiopathological" risk. However, it is a major dimension for clinicians managing people with SCI. This explains the low statistical value of scales evaluating the risk of PU in the specific SCI population [22]. These scales are useful to detect persons at risk within a heterogeneous population, but as soon as the population becomes homogenous in its pathological profile, factors directly related to the specificity of the pathology emerge.

The internal consistency of the scale was excellent, evaluated at 0.907 on the same sample of 132 people with SCI. It is comparable with the study conducted by the team who designed the original English version of the SMnac [9, 10]. Furthermore, we showed that three items reduced the internal consistency of the scale, bringing up the possibility that they did not really assess the same concept as the other items. In light of the excellent reproducibility, the high level of internal consistency of the scale including these items and their clinical relevance, we did not wish to modify the scale. The lower score in the category "preventing the onset of wound" can probably be explained by the diversity of skin lesions that were listed in this category (burn, ingrown nail, etc.).

Several limits can be brought up for this study. Some hypotheses formulated in the evaluation bear a low theoretical value, such as the relationship between coping and self-management, which was mostly brought up in qualitative studies [25]. These experimental hypotheses were only based on clinical experience but not on validated psychosocial theories, limiting the relevance of these hypotheses for the construct validity study.

Further studies are needed to reinforce the validity or assess the responsiveness of the revised SMnac scale. A factor analysis could allow the verification of the dimensions suggested by the authors. On the other hand, the predictive value of the SMnac in regards to the onset of a PU deserves further studies, in parallel to other factors known to be risk factors to PU onset in this population [30].

The revised SMnac scale is a simple, fast, reproducible, and valid tool to assess the knowledge and prevention behaviors reported by people with SCI in regards to PU. Further studies are needed to assess its responsiveness to change.

Acknowledgements We would like to thank the Fondation Paul Bennetot for its financial support, Mrs Sandrine Alonso for her precious help with database management, Mr Farid Khial, and Mrs Bénédicte Clément for the translation.

The French SMNAC Group Sarah Mathieu (Centre de Rééducation Fonctionnelle de Kerpape, Ploemeur, France), Toufik Bouchema (Centre de Rééducation Bouffard Vercelli, Cerbere, France)

Funding This study was funded by the Paul Bennetot foundation.

Author contributions GA supervised the design of the protocol, conducted the search, included patients, and wrote the manuscript,
DA was involved in the design of the protocol, conducted the search, included patients, participated to the elaboration of the manuscript and reviewed the final manuscript, DJP was in charge of statistics, GD included patients, conducted the search, reviewed the manuscripts, ME included patients, conducted the search, reviewed the manuscripts, EP included patients, conducted the search, reviewed the manuscripts, KP author of the first scale, reviewed the different version, was involved in the writing of the manuscript and final editing, FC participated to the design of the protocol, MS conducted the search and included patients, and BT conducted the search and included patients.

\section{Compliance with ethical standards}

Conflict of interest The authors declare that they have no conflict of interest.

\section{References}

1. Chen Y, DeVivo MJ, Jackson AB. Pressure ulcer prevalence in people with spinal cord injury: age-period-duration effects. Arch Phys Med Rehabil. 2005;86:1208-13.

2. Atkinson RA, Cullum NA. Interventions for pressure ulcers: a summary of evidence for prevention and treatment. Spinal Cord. 2018;56:186-98.

3. Schubart JR, Hilgart M, Lyder C. Pressure ulcer prevention and management in spinal cord-injured adults: analysis of educational needs. Adv Skin Wound Care. 2008;21:322-9.

4. Young W. Neurorehabilitation of spinal cord injury. Neurorehabil Neural Repair. 1994;8:3-9.

5. Gelis A, Pariel S, Colin D, Barrois B, Passadori Y, Ribinik P, et al. What is the role of TPE in management of patients at risk or with pressure ulcer as of 2012? Developing French guidelines for clinical practice. Ann Phys Rehabil Med. 2012;55:517-29.

6. Garber SL, Rintala DH, Holmes SA, Rodriguez GP, Freidman J. A structured educational model to improve pressure ulcer prevention knowledge in veterans with spinal cord dysfunction. J Rehabil Res Dev. 2002;39:575.

7. Rintala DH, Garber SL, Friedman JD, Holmes SA. Preventing recurrent pressure ulcers in veterans with spinal cord injury: Impact of a structured education and follow-up intervention. Arch Phys Med Rehabil. 2008;89:1429-41.

8. Berry C, Kennedy P, Hindson LM. Internal consistency and responsiveness of the Skin Management Needs Assessment Checklist post-spinal cord injury. J Spinal Cord Med. 2004;27:63-71.

9. Berry C, Kennedy P. A psychometric analysis of the Needs Assessment Checklist (NAC). Spinal Cord. 2003;41:490-501.

10. Kennedy P, Hamilton LR. The needs assessment checklist: a clinical approach to measuring outcome. Spinal Cord. 1999;37:136-9.

11. Gélis A, Daures JP, Benaim C, Kennedy P, Albert T, Colin D, et al. Evaluating self-reported pressure ulcer prevention measures in persons with spinal cord injury using the revised Skin Management Needs Assessment Checklist: reliability study. Spinal Cord. 2011;49:653-8.

12. Poitrenaud J, Moreaux C. Rapports entre performances intellectuelles et maintien d'une activité professionnelle à un âge avancé. Popul Fr Ed. 1976;31:607-16.

13. National Pressure Ulcer Advisory Panel, European Pressure Ulcer Advisory Panel and Pan Pacific, Pressure Injury Alliance. Prevention and Treatment of Pressure Ulcers: Quick Reference Guide. (ed. Emily Haesler). (Cambridge Media: Perth, Australia, 2014). 
14. Vermillion M, Dodder RA. An examination of the Rosenberg Self-Esteem Scale using collegiate wheelchair basketball student athletes. Percept Mot Skills. 2007;104:416-8.

15. Vallieres EF, Vallerand RJ. Traduction et validation canadiennefrançaise de l'échelle de l'estime de soi de rosenberg. Int J Psychol. 1990;25:305-16.

16. Cousson-Gélie F, Cosnefroy O, Christophe V, Segrestan-Crouzet C, Merckaert I, Fournier E, et al. The Ways of Coping Checklist (WCC): validation in French-speaking cancer patients. J Health Psychol. 2010;15:1246-56.

17. Zigmond AS, Snaith RP. The hospital anxiety and depression scale. Acta Psychiatr Scand. 1983;67:361-70.

18. Bjelland I, Dahl AA, Haug TT, Neckelmann D. The validity of the Hospital Anxiety and Depression Scale. An updated literature review. J Psychosom Res. 2002;52:69-77.

19. Woolrich RA, Kennedy P, Tasiemski T. A preliminary psychometric evaluation of the Hospital Anxiety and Depression Scale (HADS) in 963 people living with a spinal cord injury. Psychol Health Med. 2006;11:80-90.

20. Bergstrom N, Braden BJ, Laguzza A, Holman V. The Braden Scale for predicting pressure sore risk. Nurs Res. 1987;36:205-10.

21. Braden BJ. The Braden Scale for predicting pressure sore risk: reflections after 25 years. Adv Skin Wound Care. 2012;25:61.

22. Mortenson WB, Miller WC, SCIRE Research Team. A review of scales for assessing the risk of developing a pressure ulcer in individuals with SCI. Spinal Cord. 2008;46:168-75.
23. Krueger H, Noonan VK, Williams D, Trenaman LM, Rivers CS. The influence of depression on physical complications in spinal cord injury: behavioral mechanisms and health-care implications. Spinal Cord. 2013;51:260-6.

24. Lourenco L, Blanes L, Salomé GM, Ferreira LM. Quality of life and self-esteem in patients with paraplegia and pressure ulcers: a controlled cross-sectional study. J Wound Care. 2014;23:331-4.

25. Munce SE, Webster F, Fehlings MG, Straus SE, Jang E, Jaglal SB. Perceived facilitators and barriers to self-management in individuals with traumatic spinal cord injury: a qualitative descriptive study. BMC Neurol. 2014;14:48.

26. Cronbach L. Coefficient alpha and the internal structure of tests. Psychometrika. 1951;16:297-334.

27. Fermanian J. [Validation of assessment scales in physical medicine and rehabilitation: how are psychometric properties determined?]. Ann Readapt Med Phys Rev Sci Soc Fr Reeduc Fonct Readapt Med Phys. 2005;48:281-7.

28. Zou KH, Tuncali K, Silverman SG. Correlation and simple linear regression. Radiology. 2003;227:617-22.

29. Streiner DL. Starting at the beginning: an introduction to coefficient alpha and internal consistency. J Pers Assess. 2003;80:99-103.

30. Gélis A, Dupeyron A, Legros P, Benaïm C, Pelissier J, Fattal C. Pressure ulcer risk factors in persons with spinal cord injury Part 2: the chronic stage. Spinal Cord. 2009;47:651-61. 